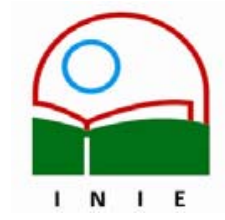

Universidad de Costa Rica

Facultad de Educación

Instituto de Investigación en Educación

ACTUALDADES INVESTIG ATIVAS EN EDUCACION

\title{
LA APROPIACIÓN DE LA LENGUA ESCRITA EN EL CICLO DE TRANSICIÓN CON BASE EN LA FILOSOFÍA DEL LENGUAJE INTEGRAL
}

\author{
THE APPROPRIATION OF THE WRITTEN LANGUAGE IN THE CYCLE OF TRANSITION \\ BASED ON THE INTEGRAL LANGUAGE PHILOSOPHY
}

\section{Rocío Carmona Mendoza ${ }^{1}$ \\ Sasha Crawford Daniels ${ }^{2}$ Ana Catalina Rivera Calvo ${ }^{3}$ \\ Ligia Zamora González ${ }^{4}$}

\begin{abstract}
Resumen: Este artículo presenta una investigación realizada sobre la apropiación de la lengua escrita en el ciclo de transición con fundamento en la filosofía del lenguaje integral.

Con base en dicha filosofía, y el referente teórico de diversos autores como Piaget, Vigotsky, Ruiz y Gómez Palacios, entre otros, se plantea una serie de estrategias por realizar en la labor docente. De esa manera estimular los cuatros procesos del lenguaje: habla, escucha, lectura y escritura. Asimismo, se presentan los resultados obtenidos en cada uno de los procesos con el grupo con el que se trabajó.
\end{abstract}

Palabras clave: PROCESOS DE ESCRITURA, PROCESOS DE LECTURA, FILOSOFÍA INTEGRAL DE LA LENGUA, ESTRATEGIAS DE ENSEÑANZA

Abstract: This article presents an investigation made on the appropriation of the written language in the cycle of transition based on the integral language philosophy.

Base in this philosophy, and the referring theoretician of diverse authors like Piaget, Vigotsky, Ruiz and Gómez Palacios, among others, considers a series of strategies to make in the educational work. Of that way to stimulate the four processes of the language: speaks, listens, reading and writing. Also, the results obtained in each one of the processes with the group appear with which it worked.

Key words: WRITING PROCESSES, READING PROCESSES, INTEGRAL LANGUAGE PHILOSOPHY, TEACHING STRATEGIES

\footnotetext{
${ }^{1}$ Licenciada en Educación Preescolar de la Universidad de Costa Rica. Actualmente labora en el Centro Educativo Santa María. Correo electrónico: rociocm@costarricense.cr

${ }^{2}$ Licenciada en Educación Preescolar de la Universidad de Costa Rica. Actualmente labora en Kamuk School. Correo electrónico: sachaquito@hotmail.com

${ }^{3}$ Licenciada en Educación Preescolar de la Universidad de Costa Rica. Actualmente labora en Koala Day kare y en el Centro de Idiomas Berlitz. Correo electrónico: acestrella@yahoo.com

${ }^{4}$ Licenciada en Educación Preescolar de la Universidad de Costa Rica. Actualmente labora en el Centro de Evaluación Académica, Universidad de Costa Rica. Correo electrónico: li_za_go@hotmail.com
}

Artículo recibido: 15 de mayo, 2006

Aprobado: 4 de diciembre, 2006 


\section{Introducción}

Se describe a continuación una investigación de tipo cualitativa que se llevó a cabo en un centro educativo público, ubicado en las cercanías de la capital de Costa Rica.

Este artículo pretende mostrar a los docentes y las docentes la importancia de trabajar en nuestros salones de clase con la filosofía del lenguaje integral como medio para adquirir la apropiación de la lengua escrita en niños y niñas del ciclo de transición.

Se han realizado varias investigaciones en torno a los procesos iniciales de la lectura y la escritura. Entre ellas, Burke, y Woodward (1982), señalan que los niños y las niñas tienen expectativas acerca del lenguaje oral para comunicarse y que esto despierta su interés en él (citado por Ruiz, 1996). Asimismo, carmen Cintrón (1996, p. 323), menciona algunos de los resultados de las investigaciones realizadas sobre la escritura en la niñez temprana, en relación con el lenguaje oral. Entre las que se encuentran las de Harste, Short y Burke (1988, pp. 51-117), y la de Morrow, (1993, pp. 112-133). En dichas investigaciones, se comprueba que el lenguaje escrito mejora a lo largo de la enseñanza de la escuela elemental, si los niños y niñas son expuestos a una literatura rica y abundante.

En nuestro contexto sociocultural, los niños y las niñas cuyas edades están entre los cero y los seis años se encuentran en continuo contacto con estímulos visuales y sonoros, al igual que con el lenguaje escrito por medio de letreros, etiquetas, envolturas de galletas, juegos, tarjetas, revistas, periódicos y otros materiales escritos que encuentran en su entorno. Hoy más que nunca, van descubriendo la importancia que tiene el lenguaje escrito para expresarse, para ser comprendidos y para comprender a los demás. En esa interacción observan que, tanto la lectura como la escritura, tienen un importante lugar en el desarrollo de la vida cotidiana de las personas, y es así como sienten la necesidad de apropiarse de dichas herramientas comunicativas para expresar lo que les gusta y piensan. Al respecto, rivera, (1999, p. 14) indica que

...cuando llegan a la escuela los niños y las niñas saben que existe la lectura y la escritura porque provienen de una cultura donde la letra impresa está presente por todas partes y tiene una diversidad de usos. Llegan con mucho conocimiento sobre la lectura y la escritura y los maestros tenemos que usar ese conocimiento para seguir estimulando el desarrollo natural del lenguaje. 
Desde esta perspectiva y con el apoyo de la investigación acción, se realizó un estudio que tenía como objetivo general promover estrategias de aprendizaje significativas que le permitiera, a la población estudiantil de un grupo del ciclo de transición, apropiarse del lenguaje escrito como herramienta de comunicación. Basada en la filosofía del lenguaje integral, la cual no es un método para la enseñanza de la lectura y de la escritura, sino una visión en el que el niño y la niña son dueños y protagonistas de su propio aprendizaje. El contexto es el campo de acción que les ofrece las oportunidades de comunicación y que les lleva al conocimiento y utilización funcional del lenguaje. En este entorno, el personal docente pasa a ser un mediador entre el niño y la niña y el objeto de estudio, que estimula y promueve ambientes placenteros para que los procesos de apropiación de la lectoescritura sea naturales y significativos.

A continuación, se presenta un resumen de los principios teóricos que orientan el estudio; el marco metodológico que presenta las diferentes fases que se trabajaron durante el proceso, las categorías y subcategorías utilizadas para el análisis, así como el resultado de las mismas y algunas reflexiones finales.

\section{Marco Teórico}

El marco teórico se dividió en tres capítulos, en el primero, se analizan los postulados de piaget y vigostky sobre cómo las personas construyen el conocimiento. El segundo, hace referencia a la apropiación de la lengua escrita como un proceso interactivo y de producción cultural, además se mencionan los niveles para la adquisición de la escritura propuestos por margarita gómez palacios y los niveles para la adquisición de la habilidad de leer propuestos por daisy ruiz. En el tercer capítulo se explica la filosofía del lenguaje integral y se mencionan diversas estrategias dirigidas a incentivar los cuatro procesos del lenguaje.

\section{A) Construcción del conocimiento, lenguaje y contexto sociocultural}

Tanto Piaget como Vigotsky, aunque presentan diferencias en sus teorías, coinciden en que el ambiente y las relaciones interpersonales juegan un papel importante en la construcción del conocimiento, por lo que las experiencias que orientan los procesos de enseñanza y aprendizaje de la lectoescritura, deben partir del contexto sociocultural del cual provienen la población de estudiantes, con el propósito de que éstas les sean significativas. 
Vigotsky plantea la teoría sociocultural, la cual indica que para comprender la psiquis y la conciencia, se debe analizar la vida de la persona y su realidad social. Para vigotsky el individuo es el resultado de la relación con su medio sociocultural. En este proceso de interacción son esenciales el uso de instrumentos: herramientas y las relaciones.

Su teoría establece que estas herramientas, a través del contacto y el aprendizaje cultural del individuo, se trasformaran en signos, es decir, se interiorizan y convierten en un medio de acción sobre sí mismo. Vigotsky (1979), citado por Chaves (2001), señala que en el desarrollo del niño y la niña, las funciones aparecen en primer lugar a nivel social, entre personas (interpsíquico) y, en segundo, a nivel individual, en el interior del individuo (intrapsíquico). A este proceso de internalización, se le llamó

ley del desarrollo psíquico (cultural), donde el principio social está sobre el principio natural biológico. Por lo tanto, las fuentes del desarrollo psíquico de la persona, no están en el sujeto mismo, sino en el sistema de sus relaciones sociales, en el sistema de su comunicación con los otros, en su actividad colectiva y conjunta con ellos. (Chaves, 2001, p. 60)

Vigotsky considera que el lenguaje se da primero en un plano social, para luego ser egocéntrico y, finalmente, interiorizado. Además, es social porque, en un principio, el lenguaje cumple una función meramente comunicativa en el plano interpsíquico; es un medio de expresión y comprensión con respecto a los otros.

Piaget, resalta que el ser humano es un ente activamente envuelto en el aprendizaje e interpretación de los eventos a su alrededor. En vez de solo responder a los estímulos, las personas actúan sobre esos estímulos, observan y aprenden de los efectos de esas mismas acciones; es decir actúan sobre su ambiente, experimentan con los elementos de la situación para observar cuáles resultados obtienen (Ormond, 1990).

Esto es el resultado de procesos opuestos (dialécticos), denominados adaptación y asimilación. Estos procesos están relacionados en cómo el individuo combina el conocimiento recién adquirido, con el ya existente en su memoria. La adaptación consiste en acomodar la nueva información a la ya existente; mientras la asimilación se refiere al cambio que se produce en el conocimiento antes adquirido, para que se pueda incorporar 
(acomodar) la información recién adquirida. Así el proceso de equilibrio mantiene un balance entre la adaptación y la asimilación.

En relación con el lenguaje, Piaget estaba interesado en la función o papel que éste juega, tanto en la vida del niño como del adulto, ya que cómo él mismo lo dice en su obra: "el lenguaje y el pensamiento del niño":

el lenguaje le sirve al individuo para comunicar su pensamiento. Pero las cosas no son tan sencillas. En primer lugar, el adulto trata, con su palabra de comunicar modos del pensamiento. Ora su lenguaje sirve para la aprobación de hechos: las palabras forman parte entonces de reflexiones objetivas, informan y permanecen ligadas al conocimiento. Por el contrario, a veces el lenguaje comunica órdenes o deseos, sirve para criticar, para amenazar; en una palabra, para despertar sentimientos y provocar actos. (Piaget, 1968, p. 17)

De acuerdo con su teoría, Piaget describe cuatro estadios de desarrollo: sensoriomotor, preoperacional, operaciones concretas y operaciones formales; cada una ofrece una edad sugerida para su inicio y término, aunque ésta puede variar de un individuo a otro. Sin embargo, como él mismo argumenta, el orden en que se presentan, es invariable. Además, su trabajo involucra el estudio del desarrollo intelectual, el rol del lenguaje y la lógica en el desarrollo cognitivo y el pensamiento abstracto.

\section{B) La apropiación de la lengua escrita como proceso constructivo, interactivo y de producción cultural.}

En el proceso de apropiación de la lengua escrita, es fundamental partir del contexto sociocultural, donde el niño y la niña están inmersos, ya que éste es el medio en que interactúa y se relaciona con los demás. En ese contexto, mediante diferentes formas, gestos, palabras, el niño y la niña expresan lo que sienten y piensan. Lo que se constituye en un aspecto importante en este proceso, en la medida que vayan adquiriendo la necesidad de comunicarse, descubrir su mundo y de socializar con los demás.

Al respecto Ferreiro (1997) indica que los procesos de leer y escribir son construcciones sociales, donde cada circunstancia histórica da nuevos sentidos a estos procesos, y en donde la madurez para la lectoescritura, dependerá mucho más de las ocasiones sociales 
que les permitan al niño y la niña, comprender cognitivamente la información que reciben de los demás, de los textos, la información obtenida en estos actos y la información específica destinada a ellos.

La adquisición de la lengua escrita es vista como un proceso en que el niño y la niña construyen mediante la interacción con su entorno y las experiencias concretas y significativas. Así los procesos de leer y escribir son construcciones sociales, que el niño y la niña van construyendo, comprendiendo y utilizando de acuerdo con sus experiencias, madurez e interés.

Gómez Palacios (1982) propone los siguientes niveles en la adquisición de la escritura:

Concreto: en este nivel, el niño y la niña no difieren dibujo de escritura. Señala las imágenes y los textos no significan nada para ellos.

Simbólico: descubre que la escritura representa algo y puede ser "leído" o interpretado. En este nivel el niño y la niña manejan varias hipótesis:

Hipótesis del nombre: el niño y la niña consideran que los textos dicen el nombre de los objetos o figuras próximas a ellos. Reconocen que dibujo y escritura están diferenciados y que los textos tienen un significado; pero para lograr identificarlo debe ir acompañado de un dibujo.

Hipótesis de cantidad: dependiendo del momento del proceso evolutivo, el niño y la niña establecerán que tiene que existir cierta cantidad de grafías para que el texto diga algo.

Hipótesis de variedad: para que un texto pueda ser leído, es necesario que los signos usados sean variados.

Lingüístico: empieza a existir una relación entre la escritura y los aspectos sonoros del habla. En este nivel, también se encuentran algunas hipótesis que el niño y la niña van planteándose:

Hipótesis silábica: la escritura está formada por tantas letras como sílabas tenga. 
Hipótesis alfabética: el niño y la niña llegan a conocer las bases del sistema alfabético de escritura y cada fonema está representado por una letra.

Asimismo, Ruiz (1999) propone 3 niveles en la adquisición de la habilidad de leer:

Concientización del lenguaje escrito en el entorno social: el niño y la niña reconocen que el lenguaje escrito que observan en su entorno, como rótulos, etiquetas y otros, comunican mensajes.

Concientización del lenguaje escrito en libros: el niño y la niña reconocen la diferencia entre el lenguaje escrito en los libros y el lenguaje oral. Identifican que un cuento tiene principio, desarrollo y final. Se desarrollan las relaciones espaciales y otras habilidades para manejar Ios libros.

Inicio de la lectura de libros: el niño y la niña utilizan las láminas como clave; parafrasea. Reconoce que son las palabras escritas las que dicen el cuento.

Cada una de estas etapas se presenta en el niño y la niña, según su interés y desarrollo biológico, sin que se den presiones por parte del adulto que esté involucrado en este proceso.

Es de gran importancia, tener conciencia de que la adquisición de la lengua escrita es un proceso en que el niño y la niña son quienes asumen el papel principal. Por lo tanto, son los que marcan el ritmo de sus niveles de internalización. Tomando en cuenta lo anterior, el y la docente deben incentivar a los estudiantes y enriquecerlos con actividades creativas, cotidianas y naturales. El y la docente tienen en sus manos la labor de propiciar de forma natural, un proceso que el niño y la niña comenzaron en sus hogares y con experiencias de sus entornos.

\section{C) La filosofía del lenguaje integral: una perspectiva diferente para aprender a leer y a escribir}

El lenguaje integral no es un método para enseñar a leer y a escribir, porque no sigue una serie de pasos, sino que es una filosofía para orientar los procesos de apropiación de la lengua escrita en el salón de clase, en la cual el niño y la niña son quienes marcan la pauta Volumen 6, Número 3, Año 2006, ISSN 1409-4703 
en la construcción del conocimiento, partiendo de sus características, necesidades e intereses y del contexto sociocultural del grupo participante (Arellano, 1997).

Así, los niños y las niñas aprenden el lenguaje como parte de una necesidad de comunicarse con otros, tomando como base, el contexto social en donde se desenvuelven. Aprenden a hablar o a escribir, partiendo de lo que ve y escucha de otros niños, niñas o adultos que están en constante interacción con ellos (Goodman, 1989).

El lenguaje oral es fácil de aprender, porque se está en contacto con él. Al igual sucede con el lenguaje escrito. Este es aprehensible cuando es significativo y forma parte del medio sociocultural al que pertenecen el niño y la niña.

Un educador o educadora que aplica el lenguaje integral toma en cuenta experiencias previas, la familia, el ambiente (tanto dentro como fuera de la escuela), para la planificación curricular y la evaluación. Dentro del trabajo en el aula busca incentivar los 4 procesos del lenguaje: habla, escucha, lectura y escritura, mediante diferentes estrategias.

\section{Metodología de la Investigación}

Se realizó una investigación cualitativa ya que, se analizan situaciones particulares, dentro de contextos particulares, por lo que no se pretende generalizar el fenómeno estudiado. En este tipo de investigación se describe, comprende, analiza y reflexiona sobre el fenómeno en estudio.

Se siguieron los principios del método de investigación - acción. Éste método presenta tres modalidades, de las cuales se escogió la investigación acción práctica (colás y buendía, 1994), la cual busca la transformación de situaciones o prácticas sociales, desde los actores mismos que participan en el proceso.

\section{A) Participantes}

El estudio se llevó a cabo en un jardín de niños ubicado en san josé participaron: una maestra del nivel de transición de educación preescolar, treinta y dos niños y niñas del grupo b con edades entre los 5 años tres meses, y los 6 años tres meses de edad, 21 familias y el equipo investigador, conformado por las autoras. 


\section{B) Fases de la investigación:}

- Elaboración teórica

- Negociación de entrada a la institución

- Sensibilización de los participantes

- Análisis del contexto sociocultural

- Diagnóstico y reconocimiento de la situación inicial

- Desarrollo del plan de acción

- Recolección de la información

- Análisis de datos

A continuación se describe cada una de las fases:

\section{Elaboración teórica}

Después de haber consultado diversas fuentes, se decidió organizar el trabajo teórico en varios apartados, con el fin de presentar una posición teórica en torno al tema por investigar y para ofrecer al lector, los aspectos que se toman en cuenta, sobre la construcción del conocimiento y el lenguaje, la apropiación de la lengua escrita y en sí lo que es la filosofía del lenguaje integral.

Negociación de entrada a la institución

En esta etapa, se negoció con la directora de la institución y al mostrarse anuente a participar dentro del proyecto, se realizó una reunión con la maestra del grupo, la directora del jardín de niños, la supervisora de la tesis y un miembro del equipo investigador, donde se explicaron los objetivos del proyecto y las implicaciones de participar en el estudio. Al principio la maestra se mostró temerosa de participar en la investigación, pero luego aceptó pensando en los beneficios que éste podría traer a la institución, a los niños y las niñas y a ella en su labor docente.

\section{Sensibilización de los participantes}

Se realizó una reunión informativa con las familias, donde se explicó la dinámica de trabajo que se llevaría a cabo en conjunto, tanto en la escuela, como en el hogar. Además se entregó una carta denominada: consentimiento informado, la cual explicaba los objetivos del proyecto y las implicaciones del mismo para las familias y los compromisos que adquirían. 
Análisis del contexto sociocultural

La institución educativa, por estar ubicada en el centro de San José, recibe una población de diferentes zonas. Entre ellas: Uruca, Pavas, Barrio México, Sabana Sur, la cual presenta diversas situaciones sociales y económicas, por lo que para conocer el contexto de donde proceden los niños y las niñas, se realizaron entrevistas a las familias. En éstas se determinó el nivel educativo, estado civil y la ocupación de los familiares con el fin de obtener una mejor visión del grupo de niños y niñas y de los hogares que participaron en el proyecto, se realizaron entrevistas para conocer su realidad socio demográfica.

\section{> Diagnóstico y reconocimiento de la situación inicial}

En esta fase, hubo un acercamiento al grupo de niños y niñas, y se utilizaron diversas estrategias que permitieron conocer el nivel de desarrollo en que se encontraban:

Prueba diagnóstica: que abarcó todas las áreas de desarrollo.

Entrevistas: con aspectos relacionados con la lectura, la escritura, escucha y habla.

Dibujos de los niños y las niñas: para determinar el nivel en que se encontraban en ese momento, de acuerdo con las etapas que menciona ruiz, en relación con los procesos iniciales de lectura.

Observación de la labor docente: donde se observó que los procesos más estimulados fueron los de habla y escucha, dejando de lado el de expresión gráfica y la interpretación de los códigos gráficos.

\section{$>$ Desarrollo del plan de acción}

El equipo investigador recopiló, elaboró, modificó e implementó prácticas que promovieron un ambiente letrado estimulador y retador para los niños y las niñas. Así como la utilización de estrategias didácticas en el aula que favorecieron la apropiación de la lengua escrita.

Algunas de estas estrategias fueron:

* Rotulación del aula en conjunto con los niños y las niñas.

* Apertura del centro de lenguaje, el cual consistió en un área específica para estimular los cuatro procesos de lenguaje. En esta área se incluyó un buzón en el cual los niños y las niñas enviaban correspondencia a sus compañeros y compañeras, familia y maestras, actividad que permitió que interiorizaran la función del lenguaje, que es la comunicación. 
* Implementación de la estrategia de la biblioteca viajera (maleta tipo ejecutivo, que contenía diverso material gráfico que los niños y las niñas se turnaban para llevar a su casa y compartir con su familia).

* Elaboración de recetas y experimentos.

* Creación de cuentos grupales.

* Narración de cuentos por medio de títeres y láminas.

* Confección de libros y periódicos.

* Canciones, trabalenguas, poesías, adivinanzas.

Como parte del trabajo con las familias, se realizaron dos talleres. Éstos tenían como objetivo, motivarlas para que utilizaran la lectura, la escritura, el habla y la escucha en sus hogares con mayor frecuencia y de forma cotidiana y creativa.

1. Primer taller: estrategias para promover la apropiación de la lengua escrita. Su objetivo era incentivar a las familias a poner en práctica estrategias para promover en sus hijos e hijas, la apropiación de la lengua escrita.

2. Segundo taller: materiales que promuevan la apropiación de la lengua escrita. Su objetivo era elaborar diferentes materiales para el aula que promovieran los cuatro procesos del lenguaje: habla, escucha, expresión gráfica y lectura.

3. Reunión final con las familias. Tenía como objetivo el compartir con las familias los logros generales del grupo durante el desarrollo del proyecto.

\section{Recolección de la información}

Cada miembro del equipo investigador observó los diferentes períodos de la jornada, pero se tomó en cuenta, sobre todo, los períodos de conversación y literatura para poner en práctica actividades relacionadas con la filosofía del lenguaje integral, las cuales permitieran cumplir con los objetivos del proyecto. Estas observaciones se llevaron a cabo durante 14 semanas, comprendidas entre los meses de marzo a junio del 2005 y como mínimo, se realizaron dos visitas por semana, éstas fueron en parejas.

Las observaciones se registraron en el diario de campo, donde cada investigadora anotó aspectos que le parecieron relevantes, como algún comentario, actitudes, diálogos, experiencias, creencias, pensamientos y reflexiones, tanto de la docente, como de los niños, niñas y las propias investigadoras. El diario se transcribió para facilitar su análisis, por lo que 
cada investigadora debía al final del día, digitarlo y realizar una interpretación de lo observado, de acuerdo con la teoría y su experiencia personal.

Para complementar las observaciones, se utilizaron grabaciones y fotografías, con el propósito de representar la realidad de una forma más concreta y contribuir con el análisis.

Además se aplicaron diversos instrumentos para obtener datos de los diferentes participantes del estudio (familia, niños, niñas y docente) tales como observaciones, entrevistas, diagnósticos.

\section{Análisis de datos}

El análisis de la información fue constante, progresivo y flexible, durante todo el proceso de estudio.

En cada reunión del equipo investigador, se daba un espacio para comentar, analizar y reflexionar sobre las experiencias en el salón de clase.

Con base en el análisis de la información recopilada y los objetivos del estudio, se propusieron las siguientes categorías y subcategorías:

1. Expresión oral: la forma en que los niños y las niñas comunican sus pensamientos, sentimientos e ideas de forma oral.

- Estructuración de oraciones: forma en que los niños y las niñas construyen oraciones.

- Interpretación personal de la expresión gráfica: es la forma cómo que ellos y ellas describen diferentes manifestaciones gráficas (rótulos, dibujos).

2. Interpretación auditiva: la percepción que puedan tener los niños y las niñas de los mensajes orales.

- Escucha: interpretación personal del lenguaje oral. 
3. Lectura: la interpretación que hacen los niños y las niñas de los códigos gráficos que encuentran en su ambiente.

- Percepción de la lectura: apreciación que tienen los niños y las niñas sobre la lectura.

- Hábitos de lectura: costumbres familiares en relación con la lectura.

- Literatura de interés: literatura que los niños y las niñas prefieren.

4. Expresión gráfica: manifestación gráfica que los niños y niñas producen.

- Percepción de la lectura: conocimiento que tienen los niños y las niñas sobre la lectura.

- Nivel de preescritura: nivel en que se ubican el niño y la niña de acuerdo con su expresión gráfica.

Con el propósito de validar la información recopilada, se triangularon los datos obtenidos en los diarios de campo de las investigadoras, con la información en las entrevistas con las familias, los niños y las niñas, la cual se contrastó a su vez con la teoría que sustentó este estudio.

\section{Resultados del Análisis}

A pesar de ser una investigación cualitativa donde el análisis de datos se hizo de forma individual, por sugerencia de una de las lectoras del presente documento, se presentaron los resultados en porcentajes para una mejor organización de la información.

\section{A. Expresión oral}

- Estructuración de oraciones.

Al inicio el proyecto se observó que un $86 \%$ de la población estructuró oraciones adecuadamente, y mostraba seguridad al expresarse oralmente. De este $86 \%$, al finalizar el estudio, un $72 \%$ no presentó cambios, mientras un $14 \%$ mejoró significativamente su expresión oral, ya que aumentó su seguridad para expresar ideas, sentimientos y pensamientos, por medio de la participación en las diferentes actividades.

El 14\% restante de la población general, presentó un avance más limitado en comparación a los demás, debido a la presencia de problemas de lenguaje, entre ellos: dificultad de 
pronunciación y articulación, problemas para comprender mensajes orales y barrera del idioma (niño extranjero).

- Interpretación de la expresión gráfica

Al inicio del estudio, un $71 \%$ de la población daba una interpretación personal del código gráfico, haciendo comentarios referidos a lo que sus dibujos y material escrito significaban, y se percibió que un $29 \%$ no hacía interpretación de la expresión gráfica, ya que aún no reconocían que estos símbolos podían transmitir un mensaje.

Al finalizar el proyecto, un 57\% continuó interpretando el material gráfico de manera adecuada, un 33\% mostró avances, ya que descubrió la naturaleza funcional y comunicativa de la expresión gráfica, y un 10\% no interiorizó este aspecto.

\section{B. Interpretación auditiva}

\section{- Escucha}

Al inicio del estudio se observó que un $86 \%$ de los niños y las niñas mostró habilidad para entender los mensajes orales que recibían, y un 14\% presentó dificultades de atención.

Al finalizar el estudio, del $86 \%$ mencionado anteriormente, un $81 \%$ se mantuvo igual, y un $5 \%$ mejoró. De los que presentaron alguna dificultad, el total evidenció un avance, ya que mostraban mayor interés a las actividades realizadas, escuchaban con mayor atención, y brindaban respuestas completas a las preguntas formuladas por las investigadoras, en dónde se promovía el pensamiento y la reflexión.

\section{Lectura}

- Percepción de la lectura

Con respecto a los niveles para la habilidad de leer, propuestos por Ruiz (1996), un 19\% pasó del primer nivel, el cual se denomina concientización del lenguaje escrito en el entorno social, en donde el niño y la niña se percatan de que el lenguaje escrito en su medio ambiente comunica mensajes (rótulos, etiquetas, entre otros), al segundo concientización del lenguaje escrito en libros, en donde el niño y la niña descubre que el lenguaje escrito en los libros suena diferente al lenguaje oral, observan que el cuento tiene principio, desarrollo y 
final, y desarrollan relaciones espaciales en los libros. Mientras que un $9 \%$ se mantuvo en el primer nivel a lo largo de la investigación sin mostrar cambios.

Asimismo, un $67 \%$ de la población participante, inició en el nivel segundo nivel de concientización del lenguaje escrito en libros, y al final del estudio pasaron al tercer nivel, el cual corresponde al de inicio de la lectura de libros. En éste comienzan a leer el cuento utilizando las láminas como clave, lee el cuento parafraseando, se percatan que son las palabras escritas y no las láminas las que "dicen" el cuento, señalan el texto del libro al leer pasando el dedo de izquierda a derecha. $Y$ un $5 \%$ no fue posible identificar en que nivel se encuentra, debido a la barrera del lenguaje, debido a que la lengua materna del niño es el chino.

- Hábitos de lectura

Al iniciar el proyecto, se observó que un $76 \%$ de la población tenía contacto frecuente con la lectura, mientras que un $24 \%$ casi no realizaba esta actividad.

La implementación de la estrategia de la biblioteca viajera, permitió que a lo largo del proyecto, diversos miembros de la familia se involucraran en la lectura del material contenido en ella, lo que fortaleció el hábito de la lectura en un 90\% y sólo en un 10\% no se observaron cambios.

\section{- Literatura de interés}

La población demostró tener mayor interés por los libros de cuentos, entre los cuales mencionaron los cuentos de princesas, fantasías, animales, aventuras. Además, un 24\% menciona los periódicos, un 19\% le agradan las revistas y un $57 \%$ no expresó tener interés por otro material diferente de los cuentos. Cabe mencionar que la mayoría de los niños y las niñas le dan importancia a la presentación del dibujo contenido en el material gráfico, como razón para elegir determinado texto.

\section{Expresión gráfica}

- Percepción de la escritura

Al inicio de la investigación, un 14\% de los niños y niñas mostró poco interés por la expresión gráfica, de éstos, al finalizar, un $9 \%$ se interesó por la misma y en un $5 \%$ no se observaron cambios. 
En cuanto al $86 \%$ de la población que mostró interés, como resultado de la implementación de las diferentes estrategias, se observó que un 62\% logró interiorizar aún más la importancia del lenguaje escrito y su función comunicativa, mientras que en un $24 \%$ no se observaron cambios.

- Nivel de preescritura

El estudio demostró que, entre los niveles de conceptualización del lenguaje escrito, un 81\% de la población pasó del nivel concreto al simbólico; un 5\% pasó del simbólico al lingüístico y un $14 \%$ se mantuvo en el nivel concreto.

Como se pudo apreciar en todas las categorías o subcategorías, hubo un 5\%. 9\% ó un 14\% de la población que presentó algún tipo de dificultad; estos corresponden a tres estudiantes, quienes presentaron dificultades en varias de las áreas del desarrollo: en la cognoscitiva, la socio-emocional y la de lenguaje.

Para fortalecer los cuatro procesos del lenguaje en ellos, se trabajó en forma individual a lo largo del proyecto, procurando, a la vez, integrarlos al grupo. Por ejemplo, al realizar las entrevistas, se les brindó más tiempo, respetando su propio ritmo, con el fin de que se sintieran cómodas en un ambiente de cordialidad. Asimismo, se les ubicó estratégicamente cerca de la investigadora que dirigía alguna actividad, para que tuvieran un mayor contacto visual y lograr una mejor atención por parte de ellos. Además, hubo un acercamiento hacia ellos por medio de pequeños diálogos espontáneos, que permitieron conocerles aún mejor.

En el caso de una de las niñas, se estaba realizando el trámite para remitirla a un especialista, quien le brindaría ayuda profesional en el área de lenguaje.

\section{Reflexiones finales}

- Contexto socio - cultural

En cuanto al nivel educativo, se observó que un alto porcentaje de los padres y las madres solamente realizó estudios de primaria y secundaria, un menor porcentaje realizó estudios universitarios. Asimismo, se evidenció que en la mayoría de los casos, los padres y madres de familia tenían una jornada de trabajo que abarcaba la mayor parte del día y principalmente se dedican a un oficio. Estos factores fueron determinantes, ya que no permitieron una participación más activa por parte de algunas familias para favorecer la 
apropiación de la lengua escrita en el hogar. A pesar de ello, el envío de la biblioteca viajera a los hogares les permitió compartir material gráfico variado, donde los niños y las niñas interactuaron con diferentes miembros de su familia.

- Plan de trabajo

Bajo el marco de la filosofía del lenguaje integral se implementaron diversas estrategias, tales como elaboración de recetas, experimentos, creación y narración de cuentos, utilizando diversos recursos como títeres, láminas, libros, proyección de películas, entre otros. Esto contribuyó a despertar el interés por la lectoescritura en los niños y las niñas, brindando un ambiente letrado de acuerdo con sus intereses, lo que les permitió adquirir conceptos y nociones sobre la lectura y la escritura. Asimismo reforzar su función comunicativa, estimulando a la vez los cuatro procesos del lenguaje: expresión oral, escucha, lectura, escritura, para lo cual, se brindaron espacios en donde los niños y las niñas pudieran expresar sus sentimientos y pensamientos acerca de diferentes temas mediante la expresión oral y gráfica.

Uno de los objetivos del plan de trabajo fue que los niños y las niñas conocieran acerca de diversos géneros literarios. Debido a lo anterior, se llevaron a cabo actividades que incluyeran adivinanzas, trabalenguas, cuentos, dramatizaciones, poesías, canciones, los cuales les abrieron un mundo de posibilidades en cuanto a gustos e intereses.

- Participación de las familias

Las familias también se mostraron interesadas con la biblioteca viajera, ya que se evidenció, a través de las entrevistas, que varios miembros de la familia participaron en la lectura del material gráfico que se incluyó en la misma. Lo anterior contribuyó a crear un ambiente de hogar que favoreciera la apropiación de la lengua escrita.

\section{Logros del plan de trabajo}

Al inicio del proyecto, se percibió que la mayoría de los niños y las niñas reconocían etiquetas y rótulos, es decir, se encontraban en la primera etapa propuesta por ruiz (1996). Ésta corresponde a la "concientización del lenguaje escrito en el entorno social"; al final del estudio se logró observar un cambio en el que la mayoría de los niños y las niñas habían avanzado hacia la etapa "inicio de la lectura de libros", donde el párvulo utiliza las láminas 
como clave, parafrasea y reconoce que son las palabras escritas las que brindan el mensaje del texto.

En cuanto a la expresión gráfica, al concluir el estudio los niños y las niñas ya incluían grafemas, por lo que se considera que pasaron de un nivel concreto, donde la escritura no difiere del dibujo, a un nivel simbólico, donde descubren que la escritura representa algo y puede ser "leído" e interpretado, esto según los niveles de gómez palacios (1982).

Por otra parte, durante el proceso de estudio, se observó que si bien la mayoría de los niños y las niñas no presentaban dificultades con la expresión oral y con la escucha, el proyecto contribuyó a fortalecer estos aspectos. Por medio de las diferentes estrategias se logró promover una mayor seguridad al expresar ideas y sentimientos mediante una adecuada construcción de frases y oraciones, al dar seguimiento entre la pregunta y la respuesta, en cualquiera de los temas tratados.

- Equipo investigador

Al iniciar el proyecto, las experiencias con respecto a los procesos de enseñanza aprendizaje acerca de la lectoescritura que tenía el equipo investigador, estaba relacionadas con métodos propuestos en las instituciones donde se había laborado, métodos que no respetaban ni tomaban en cuenta el interés natural de los niños y las niñas por la lectura y la escritura. Éstos consistían en la repetición de sílabas o fonemas que no tenían sentido para el niño y la niña, pero que si cumplen con el objetivo de enseñarles a leer y escribir de manera mecánica, no así, a apreciar y comprender la lectura.

La filosofía del lenguaje integral permite, en cambio, que el niño y la niña descubran de forma natural el mundo letrado que les rodea y se interesen por conquistarlo, siendo éste el objetivo principal de la investigación. Durante el proceso se percibió la importancia de la aplicación de esta filosofía para lograr un verdadero desarrollo integral, ya que las actividades permitieron estimular las diferentes áreas (cognoscitiva, psicomotriz, socioemocional y lenguaje) permitiendo un aprendizaje significado que brindó la oportunidad al niño y la niña de construir su conocimiento al crear, investigar, solucionar problemas y disfrutar. 
Es por esto, que al finalizar el proyecto la visión del equipo investigador ha cambiado, considerando aplicar en sus lugares de trabajo la filosofía del lenguaje integral, para orientar su labor educativa.

\section{Referencias}

Aguilar, Marcela. (2000). El lenguaje integral, una experiencia con niños en riesgo social. Tesis de Licenciatura en Educación Primaria. Universidad de Costa Rica, San José, Costa Rica.

Arellano, Adelina. (1994). El lenguaje integral y la lectoescritura en la escuela primaria latinoamericana. En Marta Eugenia Sánchez y Luz Emilia Flores (comp.), Los procesos de lectura y escritura. Selección de lecturas (pp. 149-159). Heredia: EUNA.

Arellano, Adelina. (1997). Lenguaje integral para leer, escribir, y aprender. Lima, Perú. Editores Libro Amigo.

Baquero, Ricardo. (1996). Vigotsky y el aprendizaje escolar. Buenos Aires: Aique Grupo Editor S.A.

Bolaños, Bolívar. (1994). Factores socioeconómicos y otros factores ambientales. En Marta Eugenia Sánchez y Luz Emilia Flores, Los procesos de lectura y escritura. Selección de lecturas (pp. 29-38). Heredia: EUNA.

Braslavsky, Berta. (1994). El maestro y el aprendizaje de la lectura. En Marta Eugenia Sánchez y Luz Emilia Flores, Los procesos de lectura y escritura. Selección de lecturas (pp. 13-19). Heredia: EUNA.

Braslavsky, Berta. (2004). La lengua escrita y los procesos de adquisición del conocimiento en una concepción sociohistórica-cultural. Recuperado el 26 de julio del 2004, de http://www.iacd.oas.org/la\%20educa\%20117/perlestein.htm.

Chaves, Ana Lupita. (2001). Implicaciones educativas de la teoría sociocultural de Vigotsky. Educación, 25 (2), 59-65.

Chaves, Ana Lupita. (2004). Los procesos iniciales de lecto-escritura desde la filosofía del lenguaje integral, teoría y práctica. Costa Rica: Editorial de la Universidad de Costa Rica.

Cintrón, Carmen. (1996). Estrategias para la lectoescritura en el salón de clases. Memoria del Segundo Congreso de las Américas sobre lectoescritura (pp. 323-328). Universidad Nacional. Dirección de extensión. Heredia: EUNA.

Colás, Ma. Pilar. y Buendía, Leonor. (1994). Investigación educativa (2a ed.). Sevilla, España: Alfar, S.A. 
Delgado, Elisa. (1999). Planeamiento didáctico en la educación preescolar. San José: EUNED.

Diccionario de la Real Academia de la Lengua Española. (2001). Recuperado en agosto 2005, de www.rae.es

Dobles, María., Zúñiga, Magaly y García, Jackeline. (2001). Investigación en educación: procesos, interacciones, construcciones. San José, Costa Rica: Editorial Universidad Estatal a Distancia (EUNED).

Ferreiro, Emilia. (1997). Alfabetización teoría y práctica. México: Siglo XXI.

Freire, Paulo. (1986). La importancia de leer y el proceso de liberación. México. Siglo XXI editores.

Gólcher, Raquel. (2004, 26 de setiembre). Educación preescolar se ha vuelto "seria". En Periódico La Nación, Sección El País, p. 6A. San José, Costa Rica.

Gómez Palacios, Margarita y Ferreiro, Emilia. (1982). Propuestas para el aprendizaje de la lengua escrita. México: SEPOEA.

Gómez Palacios, Margarita. (1993). Indicadores de la compresión lectora. Washington, Estados Unidos de América: Interamer.

Goodman, Kenneth. (1989). Lenguaje integral. Mérida, Venezuela: Editorial Venezolana.

Guerra, Cristina. (1999). En sus marcas...listos, todos a leer. En Al son de los tiempos: procesos y prácticas de la lectoescritura. Puerto Rico: Editora Centenario S.A.

Hernández, José. (1996). Contextos socioeducativos culturales del desarrollo reflexivo en lectoescritura. Memoria del Segundo Congreso de las Américas sobre lectoescritura (pp. 31-38). Heredia, Costa Rica: Universidad Nacional.

Hernández, Tania.; Matamoros, Kathia.; Matamoros, Vanessa.; Prialé, Victoria; Ramírez, Marjorie. (1997). La técnica del portafolio como un recurso de evaluación en el área de matemática en niños de edad preescolar. Tesis para optar por el título de Licenciatura en Educación Preescolar, Universidad de Costa Rica, San José, Costa Rica.

Instituto de Investigación para el Mejoramiento de la Educación Costarricense. (1999). Paradigma cualitativo: caracterización. San José, Costa Rica: El Instituto.

Lewis, David. (1980). Lenguaje secreto del niño. España: Editores Martínez Roca S. A.

Mayer, Richard. (1987). Educational psychology. Boston: Little, Brown and Company.

Méndez, Zayra. (2000). Aprendizaje y cognición. San José: EUNED.

Jardín de niños Juan Mora Porras. (2002). San José, Costa Rica: Documento mimeografiado. 
Ministerio de Educación Pública. (2004). Relanzamiento de la educación costarricense: pronunciamiento del departamento de educación preescolar. San José, Costa Rica: El Ministerio.

Molina, Ángeles. (1999). Precursores tempranos de la lectoescritura convencional en la infancia y en los años preescolares. Revista de Ciencia Sociales, 7. Centro de Investigaciones Sociales. Universidad de Puerto Rico. Recinto de Río Piedras. Puerto Rico.

Müller, Marta Virginia. (1993). Técnicas de comunicación oral. San José: Editorial de la Universidad de Costa Rica.

Ormrod, Jeanne Ellis. (1990). Human learning: principles, theories, and educational applications. Ohio, Estados Unidos: Merrill Publishing Company, Columbus.

Piaget, Jean. (1968). El lenguaje y pensamiento del niño. Buenos Aires: Editorial Guadalupe.

Rivera, Diana. (1999). Un nuevo acercamiento a la alfabetización: el lenguaje integral. El proceso de aprender a leer. En Al son de los tiempos: procesos y prácticas de la lectoescritura (9-33). Puerto Rico: Editora Centenario S.A.

Rivera, María Elena. (1986). El concepto de corrección y prestigio lingüísticos. México: Editorial Trillas.

Rivera, Diana, Santiago, Laura y Reyes Ibet. (1996). Nuevos acercamientos a la evaluación de los procesos iniciales de la lectoescritura en alumnos de nivel preescolar y primer grado: la experiencia de maestras puertorriqueñas ( $\mathrm{pp}$. 255259). En Memoria del Segundo Congreso de las Américas sobre lectoescritura. Universidad nacional, Dirección de Extensión. Heredia: EUNA

Riviere, Ángel. (1988). La psicología de Vigotsky. Madrid: Visor Distribuciones.

Ruiz, Daisy. (1996). La alfabetización temprana en el ambiente preescolar. San Juan, Puerto Rico: Facultad de Educación, Universidad de Puerto Rico.

Sáez, Ruth. (1999). Yo me meto al cuento y ayudo: la creación de mundos a través de la lectura. En Al son de los tiempos: procesos y prácticas de la lectoescritura (5984). Puerto Rico: Editora centenario S.A.

Sáez, Ruth; López, Laura y Santiago, Laura. (1996). El lenguaje integral y la integración curricular: la experiencia de un kindergarten puertorriqueño (pp. 339-346). Memoria del Segundo Congreso de las Américas sobre lectoescritura. Universidad nacional. Dirección de extensión. Heredia: EUNA.

Sánchez, Grettel. (2002). La filosofía del lenguaje integral: una opción para la iniciación de la lectoescritura con niños de cuatro años y seis meses a cinco años y seis meses del Colegio Teresiano San Enrique de Ossó. Tesis de optar por el grado de Licenciatura en Preescolar. Universidad de Costa Rica, San José, Costa Rica. 
Scheffler, Israel. (1973). Las condiciones del conocimiento. México D.F.: Universidad Nacional Autónoma de México. 